\title{
Challenges with Current Wearable Technology in Monitoring Health Data and Providing Positive Behavioural Support
}

\author{
Katrin Hänsel*, Natalie Wilde*, Hamed Haddadi* ${ }^{*}$, Akram Alomainy* \\ *Queen Mary University of London, UK \\ ${ }^{\dagger}$ Qatar Computing Research Institute, Qatar \\ $\{k . h a n s e l, n . w i l d e\} @ q m u l . a c . u k$
}

\begin{abstract}
There is a current trend of wearable sensing with regards to health. Wearable sensors and devices allow us to monitor various aspects of our lives. Through this monitoring, wearable systems can utilise data to positively influence an individual's overall health and wellbeing. We envisage a future where technology can effectively help us to become fitter and healthier, but the current state of wearables and future directions are unclear. In this paper, we present an overview of current methods used within wearable applications to monitor and support positive health and wellbeing within an individual. We then highlight issues and challenges outlined by previous studies and describe the future focuses of work.
\end{abstract}

\section{General Terms}

Human Factors

\section{Categories and Subject Descriptors}

H.5.m [Information Interfaces And Presentation]: Miscellaneous; J.3 [Computer Applications]: Life And Medical Sciences-Consumer Health

\section{Keywords}

wearable technology, digital health, personal informatics, big data

\section{INTRODUCTION}

In the last few years computational power has increased, storage space prices have dropped and electronic components have miniaturised. This has helped to create a new types of health and wellbeing wearable devices, allowing us to continuously monitor our own behaviours and gain insight upon ourselves from the data collected. Wearables differ in how they handle this self-made health data. Some will simply present the data back to the user for their own analysis and self-motivations. Other devices aim to contextualise data to make it more meaningful to the user and hopefully encourage positive behaviour change. But health data can benefit more than just one user, big data analysis of health data from a larger population can lead to greater insights in the human health. This can support general research in the area of health and fitness overall.

\section{BACKGROUND}

Consumer wearable technology has a strong focus on health and fitness [12]. The increased availability and ability to monitor physiological signals allow us to gain closer insights in our health. It also enables the use of new intervention techniques to influence our behaviour. Even smartwatches like the new Apple Watch, whose main purpose is not fitness tracking, are equipped with biosensors to monitor heart rate and steps made over the day. This reflects the strong interest of individuals in wanting to know about their own health and fitness.

The variety of 'smart' consumer devices for health and wellbeing monitoring range from general devices like activity trackers, body weight and sleep monitors,${ }^{1}$ to fitness performance devices like smart socks to improve running style.

2 There are even medical devices for measuring blood glucose or blood pressure like the products from iHealth. ${ }^{3}$ Their ability to connect to other devices and the Internet of Things to share data has earned them the name 'smart' and distinguishes them from old-school, non-connected step counters, wristwatches or garments.

For health sensors, the enabled synchronisation of data eases the burden of manually keeping track of data and provides better accessibility for analysis. The synchronised data can usually be accessed via a web interface in a prepared and visualised form, the raw data is hidden from the user. Already available APIs provide third-party access and platforms to collect this personal health data [29].

\section{Quantified Self and Internet-Informed Patient}

The sensing of data has already found its way into doctor's practises. The three big mobile phone operating system companies have reacted to the increased consumer interest

\footnotetext{
${ }^{1}$ e.g. Fitbit's products www.fitbit.com

2 www . sensoriafitness. com

${ }^{3}$ www . ihealthlabs.com
} 
by integrating health data storage into their ecosystem. Microsoft's Health Vault started as a Personal Health Record (PHR) system with a focus on fitness and medical conditions which could be shared with a medical professional [14]. PHRs proved to be effective and powerful tools for personal health management and are an electronic interface to the treating physician [25]. There is a shift from the patient being passive to becoming an 'Internet Informed' and participatory patient [21]. This includes patients who monitor their health and share data with the physician. But with these new patient-enabling technologies, the risk of false selfdiagnosis is rising [27]. People with health anxiety can spend hours using the internet to check for health symptoms, this is known as Cyberchondria [23].

Apple's HealthKit and Google Fit are new mobile healthtracking platforms with a focus on fitness and wellbeing. They allow the storage and interchange of health data between different applications. This makes techniques used by the Quantified Self (QS) community accessible to a broader population. The QS trend describes the quantification of aspects within our lives in order to allow us to reflect, learn and improve by collecting data and analysing it. Automated recording of these aspects can ease this process and analysis and is an important factor in preventing tracking fatigue and failure [10]. The majority of self quantifiers are professionals, software engineers or data analysts [10] with the necessary skills to analyse the gathered data. Appropriate applications and tools for data aggregation and automated analysis could support people without these skills to gain insights in their data. Meaningful feedback which is adapted to the user's needs is a key to success.

\section{Making Data Meaningful}

Data needs to be meaningful to the user in order to support their behaviours over a long period of time. Experts suggest that displaying a large amount of raw data and a lot of numbers can lead to the user becoming discouraged [15]. In fact, most patients equipped with pedometers lose interest in the data after a period of time [5]. Wearable applications currently use a number of methods to avoid just presenting raw data to the user, preventing cognitive overload from processing lots of information. Ambient feedback, a form of subtle and unobtrusive feedback that is easier for the user to process, can increase the perception of the sensing data [19]. Studies have shown the use of ambient displays to be more persuasive on human behaviour than displaying numerical values because they use less cognitive resources and do not require the user's conscious attention [16].

Ubifit is a mobile application that includes an ambient, glancable display which shows a non-literal representation of the physical activity that the user has done [11]. The display contains a metaphor of a garden; the user gains more flowers by exercising more. Data is collected from sensors on the user's body and analysed to change the aesthetics of the garden throughout the week. In the study, participants agreed that the metaphorical representation of their data helped them to focus on and work towards their goals.

Gamification and social incentives can be other helpful and motivational tools. Gamification is the application of gaming elements within non-game contexts which includes leader boards, point scoring and rewards. World of Workout is a game developed to reduce sedentary lifestyles in college students by rewarding step count goals achieved [13].
Social incentives include multi-player games which utilise health data to suit either competitive or collaborative modes of play. Into merges together multiple players step count data to complete quests for rewards [2]. These games hide the complex sensing data and present them using gaming metaphors.

Contextualising information from wearables and generating smart interventions have the potential to support positive behaviour change. Just-in-time messaging and the display of data at important decision times has been proven to be effective in supporting behaviour change [18]. Smart and context-aware algorithms are able to tell what the user is doing from obtaining sensor data. Motivational suggestions can then be made at specific, influential times in the day. Step up life provides suggestions of small, suitable activities at regular intervals in the form of on-screen nudge reminders [26]. Recognising and responding at times where the user needs more support can help to keep them on track.

Personalisation is another way of supporting long-term engagement with wearable devices. Users prefer to be able to create their own interfaces to display data [5]. They do not like using a predefined default interfaces because it does not feel personal. Work by Ananthanarayan et al. focusses upon the effectiveness of self-crafted devices [4]. The wearable device included a set of sensors that tracked an individual's UV exposure and gave feedback via coloured LEDs. The participants were instructed to create their own cases to accommodate the sensors. Allowing the users to craft their own case lead to the device being accepted easily.

Some wearable applications are utilising data in a way that draws upon cognitive theories. Ajzen created the 'Theory of Planned Behaviour' (TPB) which states that intentions are the best predictor of how an individual is going to behave in certain situations [3] (see Figure 1). Three factors produce these intentions: attitude towards the behaviour, subjective norms (whether others approve or perform the behaviour) and perceived behavioural control (the extent to which the individual believes that they can carry out the behaviour). There is an indication that TPB in mobile sensing apps can support physical activity in teenagers [6]. Self efficacy is related to the TPB and describes a person's belief in their ability to succeed within a specific situation. These beliefs are great drivers of how people think, behave and feel [7]. A person with a strong sense of self efficacy forms commitment to tasks and likes to master challenges while a weak sense of self efficacy would lead to avoidance of challenging tasks altogether. In order to support an individual's behaviour in the long term, their self efficacy needs to remain high. Wearables can support this by facilitating achievable goals. Moller et al. created the GymSkill in [22], a mobile application which analyses sensor data to present the user with goals suitable for their ability. In testing users liked the personalised feedback and support.

Lifelogs record various aspects of a person's lifestyle to encourage self-reflection. Affective Diary is a digital diary that accesses stress data from sensors and mobile usage and presents it with photos taken to make it meaningful [28]. Studies found that some users appreciated the application as it helped their self-understanding. Feedback can also get a user to reflect upon the future consequences of their behaviours. FatBelt is a waist worn device which uses physical feedback by inflating around the user's stomach when too many calories are consumed [24]. This simulates the long- 


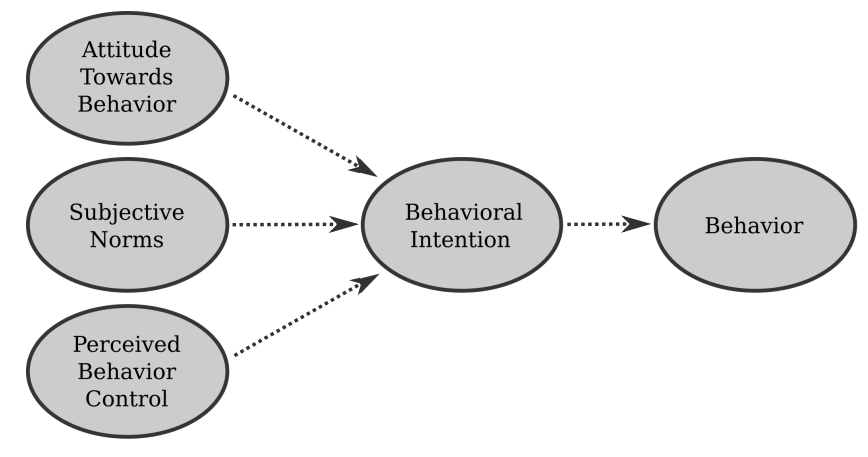

Figure 1: Theory of Planned Behaviour - The three factors influencing our intention for behaviour and the behavioural outcome: attitude towards the behaviour; subjective norm and the perception of behaviour influenced by others; perceived behavioural control and ability to master the challenge

term weight gain associated with overeating. The inflation of the device supported a significant decrease in calorie consumption. Overall, the key factor of the self-monitoring and reflection is the ability to make sense of data.

\section{Big Data for Health}

The most recent June ' 15 issue of the IEEE Spectrum journal is dedicated to the 'Hacking of the Human OS' [1] and shows how 'hot' this topic is right now. It is focused on what we can learn from utilising large amounts of data about our own health. There is a high potential for using big data the collection, storage and analysis of large, complex data sets - to learn about our health with new miniature, wearable health sensors playing a major role. Barrett et al. see the high potential in new embedded sensing technologies which enable the collection of health relevant data at a larger scale [8]. Big data can help to promote health and prevent disease by identifying determinants of health and suitable interventions for healthier behaviour.

The emerging Internet of Things (IoT) trend, which is the process of connected real world objects to the internet, can support this by opening up new data sources like health wearables, connected and ubiquitous devices, or environmental sensors. This can provide insights in complex dependencies between health, wellbeing and our environment [29]. Furthermore, they can act as outlets for smart, persuasive feedback to the user.

Apple's ResearchKit is a research focused interface to access large scale health data of consenting patients, making study participation widely accessible. One of the first examples of a broadly available study using big health data from mobile phones is a project which utilises Apple's ResearchKit for an mobile asthma health study [17]. It also utilises environmental data like air pollution.

We agree that there is high potential in learning about complex health and wellbeing correlations by having access to various health markers of a large population over a long time. But there are challenges and guidelines that have to be followed. Ethical and privacy concerns are very important when it comes to the sensitive health data. It is essential to inform patients on how their data is being handled and give them an understanding of the exact role that they are playing and the mechanisms involved.

\section{FUTURE WORK AND CHALLENGES}

As proven in the above presented research, wearable technology can have a positive impact in encouraging healthy behaviours. With the increase of consumer wearables, we now face the challenge to provide health promoting applications for these devices. These applications need to address such problems as long-term engagement, contextualisation and individualisation to provide optimal support for users with different needs and abilities.

Studies have shown that wearable applications can have clear benefits compared to mobile apps, due to an increased availability of the device [26]. But there is still further work required on making them more adaptable to different settings and lifestyles. Future devices should support a person's lifestyle and be able to adapt to each user instead of the users having to compromise their behaviours. By ensuring this, it may lead to long-term retention of future wearable devices.

We believe that no wearable application can fit the needs of every user and different levels of support are required. User groups such as the elderly may need a different type of support to help trigger health behaviour changes. Interdisciplinary work between engineers and psychologists can ensure that both the technological aspects and expertise on behaviour change techniques are considered in the design of new applications.

One approach of creating a device that is less intrusive is to allow the user to craft and personalise their own devices [4]. Further work in this area will look at creating totally modular devices, allowing the user to choose exactly what form they would prefer. The creators of Ubifit also highlight the need of creating devices that blend into the environment of the wearer with reference to sports activities [11].

As well as environment and lifestyle, future work may also focus on creating devices that allow for better social integration. The World of Workout creators stress the potential of utilising an individual's social networks. They are basing their future work around online social network integration to allow players to challenge others online [13]. Modern gamification techniques are often applied to promote both exercise and fun through exergames. The motivation to engage in the exercise arises within the individual because they enjoy the behaviour and it is rewarding for them [9]. But it is not clear whether the user enjoys the exercise or the gaming elements. Related research suggests that the removal of game elements can lead to a decreased use of the system [30]. It is promising to investigate gamification techniques for promoting healthy habits which can last without the need for gaming triggers.

Future work needs to focus on ensuring the long-term usage of wearable devices. There is a proven novelty factor when it comes to health data as stated in [5], but long-term retention has to be ensured. For example, the creators of the FatBelt have realised the device's potential but are now focussing efforts on making the device effective for the wearer when used for a longer period of time.

Changes in the healthcare sector have to address the shift to an empowered patient and the potential of wearable sensing data to personalise health treatment. These changes affect different levels of the healthcare sector from regulatory bodies as well as work flows in medical practices [20]. Modern healthcare and medical professionals need training and support to react to this trend. This includes address- 
ing patients' requests to review large amounts of healthtracking data, making suitable recommendations for their self-monitoring and pointing to reliable online information sources. There is still room for research on the effect of the availability of one's bio-physiological data and the risk of an 'obsession' and over-interpretation of this data. Selfdiagnosis without the medical knowledge or sensor accuracy can set the user on a dangerous path and needs to be considered in the design for wearable applications.

\section{CONCLUSION}

We discussed current trends in wearable technology applications and their efforts to promote positive health behaviour. We showed that the future of wearable application not merely lies in the development of new analysis algorithms for health data but also in the support of the user to make sense of data and get contextualised feedback for behaviour change. Interdisciplinary work between medical professionals, engineers and psychologists is key for the success of the new emerging wearable applications.

\section{REFERENCES}

[1] Hacking the human os. Spectrum, IEEE, 52(6), June 2015.

[2] A. Ahtinen, P. Huuskonen, and J. Häkkilä. Let's all get up and walk to the north pole: design and evaluation of a mobile wellness application. In Proceedings of the 6th Nordic Conference on Human-Computer Interaction: Extending Boundaries, pages 3-12. ACM, 2010.

[3] I. Ajzen. The theory of planned behavior. Organizational behavior and human decision processes, 50(2):179-211, 1991.

[4] S. Ananthanarayan, N. Lapinski, K. Siek, and M. Eisenberg. Towards the crafting of personal health technologies. In Proceedings of the 2014 conference on Designing interactive systems, pages 587-596. ACM, 2014.

[5] S. Ananthanarayan and K. A. Siek. Persuasive wearable technology design for health and wellness. In Pervasive Computing Technologies for Healthcare (PervasiveHealth), 2012 6th International Conference on, pages 236-240. IEEE, 2012.

[6] S. M. Arteaga, M. Kudeki, A. Woodworth, and S. Kurniawan. Mobile system to motivate teenagers' physical activity. pages 1-10, 2010.

[7] A. Bandura. Self-efficacy: toward a unifying theory of behavioral change. Psychological review, 84(2):191, 1977.

[8] M. A. Barrett, O. Humblet, R. A. Hiatt, and N. E. Adler. Big Data and Disease Prevention: From Quantified Self to Quantified Communities. Big Data, 1(3):168-175, Sept. 2013.

[9] K. Cherry. What is intrinsic motivation? www.psychology . about.com/od/motivation/f/intrinsic-motivation.htm. Accessed: 14-04-2015.

[10] E. K. Choe, N. B. Lee, B. Lee, W. Pratt, and J. A. Kientz. Understanding quantified-selfers' practices in collecting and exploring personal data. In Proceedings of the SIGCHI Conference on Human Factors in Computing Systems, pages 1143-1152. ACM, 2014.

[11] S. Consolvo, D. W. McDonald, T. Toscos, M. Y. Chen, J. Froehlich, B. Harrison, P. Klasnja, A. LaMarca, L. LeGrand, R. Libby, et al. Activity sensing in the wild: a field trial of ubifit garden. In Proceedings of the SIGCHI Conference on Human Factors in Computing Systems, pages 1797-1806. ACM, 2008.

[12] T. Danova. The Wearables Report: Growth trends, consumer attitudes, and why smartwatches will dominate. http://uk. businessinsider.com/ the-wearable-computing-market-report-2014-10, May 2015. Accessed: 28-06-2015.
[13] K. Doran, S. Pickford, C. Austin, and T. W. andT. Barnes. World of workout: Towards pervasive, intrinsically motivated, mobile exergaming. Meaningful Play Conference, 2010.

[14] L. Fernandez-Luque, R. Karlsen, T. Krogstad, T. Burkow, and L. Vognild. Personalized health applications in the web 2.0: The emergence of a new approach. In Proceedings of the IEEE on Engineering in Medicine and Biology Society, pages 1053-1056, Aug 2010.

[15] R. Gockley, M. Marotta, C. Rogoff, and A. Tang. Aviva: a health and fitness monitor for young women. In CHI'06 Extended Abstracts on Human Factors in Computing Systems, pages 1819-1824. ACM, 2006.

[16] J. Ham and C. Midden. Ambient persuasive technology needs little cognitive effort: the differential effects of cognitive load on lighting feedback versus factual feedback. In Persuasive Technology, pages 132-142. Springer, 2010.

[17] Icahn School of Medicine. Asthma mobile health study. http://apps.icahn.mssm.edu/asthma/, 2015. Accessed: 28-06-2015.

[18] S. S. Intille. A new research challenge: persuasive technology to motivate healthy aging. IEEE Transactions on Information Technology in Biomedicine, 8(3):235-237, 2004.

[19] S. Maan, B. Merkus, J. Ham, and C. Midden. Making it not too obvious: the effect of ambient light feedback on space heating energy consumption. Energy Efficiency, 4(2):175-183, 2011.

[20] M. D. Majmudar, L. A. Colucci, and A. B. Landman. The quantified patient of the future: Opportunities and challenges. Healthcare, Mar. 2015.

[21] M. McMullan. Patients using the Internet to obtain health information: How this affects the patient-health professional relationship. Patient Education and Counseling, 63(1):24-28, Jan. 2006.

[22] A. Möller, L. Roalter, S. Diewald, J. Scherr, M. Kranz, N. Hammerla, P. Olivier, and T. Plötz. Gymskill: A personal trainer for physical exercises. In Proceedings of the IEEE International Conference on Pervasive Computing and Communications, pages 213-220. IEEE, 2012.

[23] K. Muse, F. McManus, C. Leung, B. Meghreblian, and J. M. G. Williams. Cyberchondriasis: Fact or fiction? A preliminary examination of the relationship between health anxiety and searching for health information on the Internet. Journal of Anxiety Disorders, 26(1):189-196, Jan. 2012.

[24] T. Pels, C. Kao, and S. Goel. Fatbelt: motivating behavior change through isomorphic feedback. In Proceedings of the adjunct publication of the 27th annual ACM symposium on User interface software and technology, pages 123-124. ACM, 2014.

[25] S. Pushpangadan and C. Seckman. Consumer Perspective on Personal Health Records: A Review of the Literature. Online Journal of Nursing Informatics, 19(1), 2015.

[26] V. Rajanna, R. Lara-Garduno, D. J. Behera, K. Madanagopal, D. Goldberg, and T. Hammond. Step up life: A context aware health assistant. pages 21-30, 2014.

[27] N. Robertson, M. Polonsky, and L. McQuilken. Are my symptoms serious Dr Google? A resource-based typology of value co-destruction in online self-diagnosis. Australasian Marketing Journal (AMJ), 22(3):246-256, Aug. 2014.

[28] A. Ståhl, K. Höök, M. Svensson, A. S. Taylor, and M. Combetto. Experiencing the Affective Diary. Personal and Ubiquitous Computing, 13(5):365-378, 2009.

[29] M. Swan. Sensor Mania! The Internet of Things, Wearable Computing, Objective Metrics, and the Quantified Self 2.0. Journal of Sensor and Actuator Networks, 1(3):217-253, Dec. 2012.

[30] J. Thom, D. Millen, and J. DiMicco. Removing gamification from an enterprise sns. In Proceedings of the ACM 2012 Conference on Computer Supported Cooperative Work, pages 1067-1070, New York, NY, USA, 2012. ACM. 